Published in final edited form as:

Nat Rev Gastroenterol Hepatol. 2014 November ; 11(11): 645-647. doi:10.1038/nrgastro.2014.157.

\title{
HCC-subtypes, stratification and sorafenib
}

\author{
Gregory J. Gores \\ Division of Gastroenterology and Hepatology, Mayo College of Medicine, Mayo Clinic, 200 First \\ Street SW, Rochester, MN 55905, USA
}

\begin{abstract}
The past 10 years have represented a whirlwind of activity with regard to information on the risk factors, aetiopathogenesis, diagnosis and treatment of hepatocellular carcinoma. I will describe what I consider to be the major advances from both a tumour biology perspective and a clinical perspective over the past 10 years.
\end{abstract}

We are beginning to obtain insights into the initiating steps of hepatocellular carcinoma (HCC) and to understand the tumour biology of this lethal malignancy. We have learnt that polymorphisms in the $E G F$ gene (encoding epidermal growth factor), which are associated with alterations in expression, increase the risk of $\mathrm{HCC}$ development in patients with cirrhosis. ${ }^{1}$ The association between $E F G$ expression and HCC risk has resulted in ongoing clinical studies using $E G F$ receptor inhibitors for chemoprevention of $\mathrm{HCC}$ in high-risk patients. Despite the tremendous heterogeneity of the genetic aberrations in advanced HCC, it is likely that high-frequency somatic mutations in the telomerase reverse-transcriptase promoter drive early oncogenesis of this neoplasm. ${ }^{2}$ In 2008, an important observation was made by examining gene expression in fixed tissue samples from patients with HCC largely due to $\mathrm{HCV}$ infection. ${ }^{3}$ A reproducible genetic expression signature from nonmalignant liver tissue in patients with $\mathrm{HCC}$ was identified, which predicted late development of recurrent HCC following hepatic resection. The nonmalignant genetic signature supports the concept that there is a hepatic field defect (the concept that cancer results from tissue alterations throughout the liver as opposed to somatic mutations within a small group of cells) in patients who develop HCC.

Although molecular profiling has yet to be useful in selecting treatment strategies for HCC, it has become increasingly useful for prognostication of patients with HCC. An HCC fivegene score, identified in 2013, was rigorously validated and associated with survival of patients after liver resection for HCC. ${ }^{4}$ The five-gene score was based on a combination expression profile of the following genes: HN1, RAN, RAMP3, KRT19 and TAF9. This profile was identified empirically and the genes are not functionally related. Disease-specific survival was predicted by combining data on microvascular invasion, the Barcelona Clinic Liver Cancer classification and the five-gene score. Thus, for the first time, we have a potential biomarker to stratify patients for various therapeutic decisions. In 2014, work suggested that KRT19 expression can be used to identify a subset of patients who have HCC

Competing interests

The author declares no competing interests. 
with a poor prognosis. ${ }^{5} \mathrm{KRT19}$ is a cholangiocyte marker, but can be expressed by a subset of HCC cells either due to cell differentiation, cell plasticity, or a common progenitor cell of origin between hepatocytes and cholangiocytes. ${ }^{5}$ Indeed, it has been suggested that the subset of carcinomas that are KRT19 positive should be considered as a separate subtype of HCC and perhaps be managed differently. ${ }^{5}$ The genetics involved in the pathogenesis of HCC and those biomarkers that portend a bad prognosis are now starting to be understood. Stratification of patients with HCC will ultimately be very important as we begin to design personalized therapies.

A prominent paper in terms of advances in the clinical management of $\mathrm{HCC}$ of the past decade was the 2006 study demonstrating that patients infected with HBV and with elevated serum HBV DNA levels were at high risk of developing HCC. ${ }^{6}$ Subsequently, a variety of studies have confirmed this observation, demonstrating that reduction of HBV DNA levels reduces the risk of $\mathrm{HCC}$ in this patient population; this finding provides additional impetus for treating selected patients who have active HBV infection with antiviral agents. Radiographic imaging has greatly improved over the past decade and it is possible to identify increasingly smaller nodules, which might or might not be early liver cell cancers. A rigorous study was published in 2008 indicating that noninvasive techniques can be used to identify patients with $\mathrm{HCC}$ between $10-20 \mathrm{~mm}$ in diameter, ${ }^{7}$ substantiating the existing practice guidelines for early diagnosis of $\mathrm{HCC}$ by noninvasive criteria. The response to locoregional therapy has been assumed to relate to overall survival. However, this concept remained more of a supposition than it did an evidence-based conclusion. Experience from an established treatment centre showed that the radiographic response to locoregional therapy does predict patient survival. ${ }^{8}$ Memon and colleague's 2011 study gave further credence for the use of locoregional therapy as treatment for patients with HCC and in monitoring the radiographic response to determine further locoregional treatment over time.

"Stratification of patients important as we begin to design with HCC will ultimately be very personalized therapies"

Liver transplantation for patients with HCC also remains an area of active interest. However, most issues relate to organ shortage and how the stewardship of this rare resource can be counterbalanced by the potential benefit to the patient. A major analysis was put forward in 2009 by the original author of the Milan criteria identifying a subgroup of patients who might do well with liver transplantation but who have cancers beyond the Milan criteria. ${ }^{9} \mathrm{~A}$ more precise estimation of survival was suggested by using the up-to-seven criteria. The total tumour diameter of up to $7 \mathrm{~cm}$ in size (for example, seven small tumours each $1 \mathrm{~cm}$ or a single lesion of $7 \mathrm{~cm}$ ) would be predicted to identify patients who would benefit substantially from liver transplantation.

The manuscript that would probably end up on everybody's list for advances in the past decade was the results of the 2008 study demonstrating a survival benefit for patients treated with sorafenib in advanced HCC. ${ }^{10}$ The paper by Llovet et al. was a landmark in so many different ways and from so many different perspectives. First, the data illustrated that the sole focus on tumour response rates might not tell the entire story. Only a few percent of the patients had classic response rates (diminution in tumour size). Rather, therapy worked by delaying progression of the disease and so time-to-event has become a major end point in 
oncological studies. Second, sorafenib was the first systemic therapy to conclusively demonstrate a survival benefit for HCC in a randomized, placebo-controlled trial. Finally, no other chemotherapeutic agent has yet matched the results obtained with sorafenib. Thus, sorafenib continues to stand alone as a therapeutic agent for HCC and we have yet to understand precisely how and why certain patients benefit from sorafenib therapy.

Looking back is easy-looking forward is more challenging, but more intriguing (Figure 1). We are beginning to understand the heterogeneity of $\mathrm{HCC}$, which clinicians should be able to use to stratify patients for clinical trials. I believe that approaches targeting specific subtypes of HCC will prove to be important, such as different therapies for those who are negative or positive for KRT19 expression. I also believe that we need further studies using immunological therapies for the treatment of $\mathrm{HCC}$ and more attention should be paid to therapies focused on the tumour microenvironment. Hopefully in another 10 years I will be asked to write a similar article as this one, which I would hope will highlight more impressive therapeutic advances.

\section{Acknowledgments}

This work was supported by National Institute of Health Grant DK59427 and the Mayo Clinic.

\section{References}

1. Tanabe KK, et al. Epidermal growth factor gene functional polymorphism and the risk of hepatocellular carcinoma in patients with cirrhosis. JAMA. 2008; 299:53-60. [PubMed: 18167406]

2. Nault JC, et al. High frequency of telomerase reverse-transcriptase promoter somatic mutations in hepatocellular carcinoma and preneoplastic lesions. Nat Commun. 2013; 4:2218. [PubMed: 23887712]

3. Hoshida Y, et al. Gene expression in fixed tissues and outcome in hepatocellular carcinoma. N Engl J Med. 2008; 359:1995-2004. [PubMed: 18923165]

4. Nault JC, et al. A hepatocellular carcinoma 5-gene score associated with survival of patients after liver resection. Gastroenterology. 2013; 145:176-187. [PubMed: 23567350]

5. Govaere O, et al. Keratin 19: a key role player in the invasion of human hepatocellular carcinomas. Gut. 2014; 63:674-685. [PubMed: 23958557]

6. Chen CJ, et al. Risk of hepatocellular carcinoma across a biological gradient of serum hepatitis B virus DNA level. JAMA. 2006; 295:65-73. [PubMed: 16391218]

7. Forner A, et al. Diagnosis of hepatic nodules $20 \mathrm{~mm}$ or smaller in cirrhosis: prospective validation of the noninvasive diagnostic criteria for hepatocellular carcinoma. Hepatology. 2008; 47:97-104. [PubMed: 18069697]

8. Memon K, et al. Radiographic response to locoregional therapy in hepatocellular carcinoma predicts patient survival times. Gastroenterology. 2011; 141:526-535. [PubMed: 21664356]

9. Mazzaferro V, et al. Predicting survival after liver transplantation in patients with hepatocellular carcinoma beyond the Milan criteria: a retrospective, exploratory analysis. Lancet Oncol. 2009; 10:35-43. [PubMed: 19058754]

10. Llovet JM, et al. Sorafenib in advanced hepatocellular carcinoma. N Engl J Med. 2008; 359:378390. [PubMed: 18650514] 


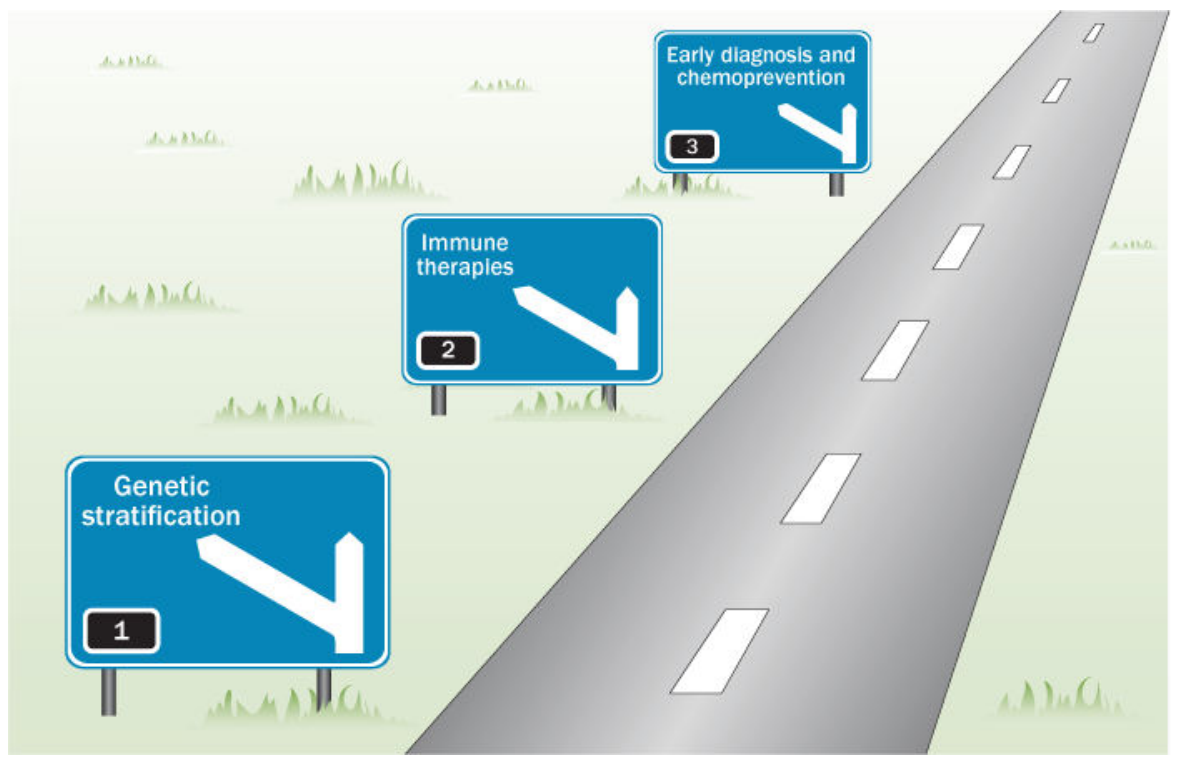

Figure 1.

Future strategies for HCC diagnosis and treatment. () Mayo Clinic. 